\title{
Le poids de l'information non financière dans les décisions de financement et d'investissement
}

\author{
Félix Zogning Nguimeya \\ Université du Québec en Outaouais
}

\section{INTRODUCTION}

\section{La dynamique actuelle des marchés rend les bailleurs de fonds (créanciers ou investisseurs) davantage friands d'informations de toutes sources, susceptibles de minimiser leurs risques ou de maximiser leur rendement à l'égard des entreprises qu'ils financent.}

La dynamique actuelle des marchés rend les bailleurs de fonds (créanciers ou investisseurs) davantage friands d'informations de toutes sources, susceptibles de minimiser leurs risques ou de maximiser leur rendement à l'égard des entreprises qu'ils financent. Lorsque ces entreprises sont

\section{MÉTHODOLOGIE}

La recherche se décline en deux méthodes. D'abord, deux entrevues ont été menées auprès d'un analyste financier et d'un courtier en valeurs mobilières, gestionnaire de placements. Le choix est porté sur ces derniers parce qu'ils font figure d'utilisateurs sophistiqués de l'information corporative et qu'ils conseillent les investisseurs. Nous assumons en filigrane que ce sont leurs choix qui s'exécutent à travers les investisseurs sur les marchés. Ces entrevues structurées ont pour but d'explorer de leur point de vue la pertinence des informations non financières dans l'évaluation qu'ils font des titres d'une entreprise. Ces analystes interviewés suivent tous les entreprises du TSX300, l'indice de la bourse de Toronto.

La seconde méthode employée est une analyse de contenu des recommandations d'analystes au sujet des titres des entreprises du TSX300. En effet, de nombreuses firmes d'analyse financière rendent publiques leurs recommandations et très souvent, leurs notes d'analyse financière, dans des journaux spécialisés de finance (Globe Money, Toronto Star, Les Affaires). En mettant un accent sur les cotées, l'information financière traditionnelle telle que présentée dans les états financiers s'avère incomplète pour jauger le potentiel de l'entreprise ou pour prédire sa santé financière à moyen et à long terme. Ainsi, toute information non financière capable de renseigner sur le potentiel latent et les perspectives de l'entreprise devient pertinente pour les offreurs de capitaux.

Cet article a pour but d'examiner dans quelle mesure les informations non financières pèsent dans les décisions des investisseurs sur les marchés financiers.

arguments avancés par les analystes dans leurs notes pour justifier leurs recommandations, nous examinerons les fréquences, occurrences et cooccurrences qui en émergent. Ceci nous éclaire $a$ priori sur le type d'informations dont ils se servent véritablement ou qui sont vraiment pertinentes pour eux. La place et la portée de l'information non financière pourra ainsi se dessiner. Les recommandations collectées dans ces journaux spécialisés de finance ont majori-tairement porté sur les entreprises du TSX300. L'analyse des recommandations déjà formulées par des analystes est par ailleurs une possibilité de corroborer les réponses obtenues lors des entrevues que nous avons menées; ce qui pourrait baliser ou corriger les limites attribuées à l'entrevue comme méthode de cueillette de données.

Ces entrevues, réalisées par enregistrement sonore, ont été transcrites pour analyse. Concernant les notes de recommandations publiées dans les journaux spécialisés, n'ont été retenus pour analyse que les commentaires ou les arguments mis en avant par les analystes pour supporter ou appuyer 
que les commentaires ou les arguments mis en avant par les analystes pour supporter ou appuyer une recommandation. Les calculs stochastiques et les graphiques techniques ont donc été isolés dans leurs publications. Deux analyses distinctes (entrevues et notes de recommandations) sont faites à l'aide du logiciel Atlas/Ti, et les résultats sont ensuite comparés. Ces analyses catégorielles tournent autour des codes suivants: Croissance, Succès financier, Stratégie et gouvernance, Conditions de marché, Structure financière, Innovation, Performance. Pour chacun de ces codes, se rapporte une liste de synonymes ou d'attributs qui pourraient y référer, comme le montre la classification suivante :

Tableau 1 - Classification des codes d'analyse

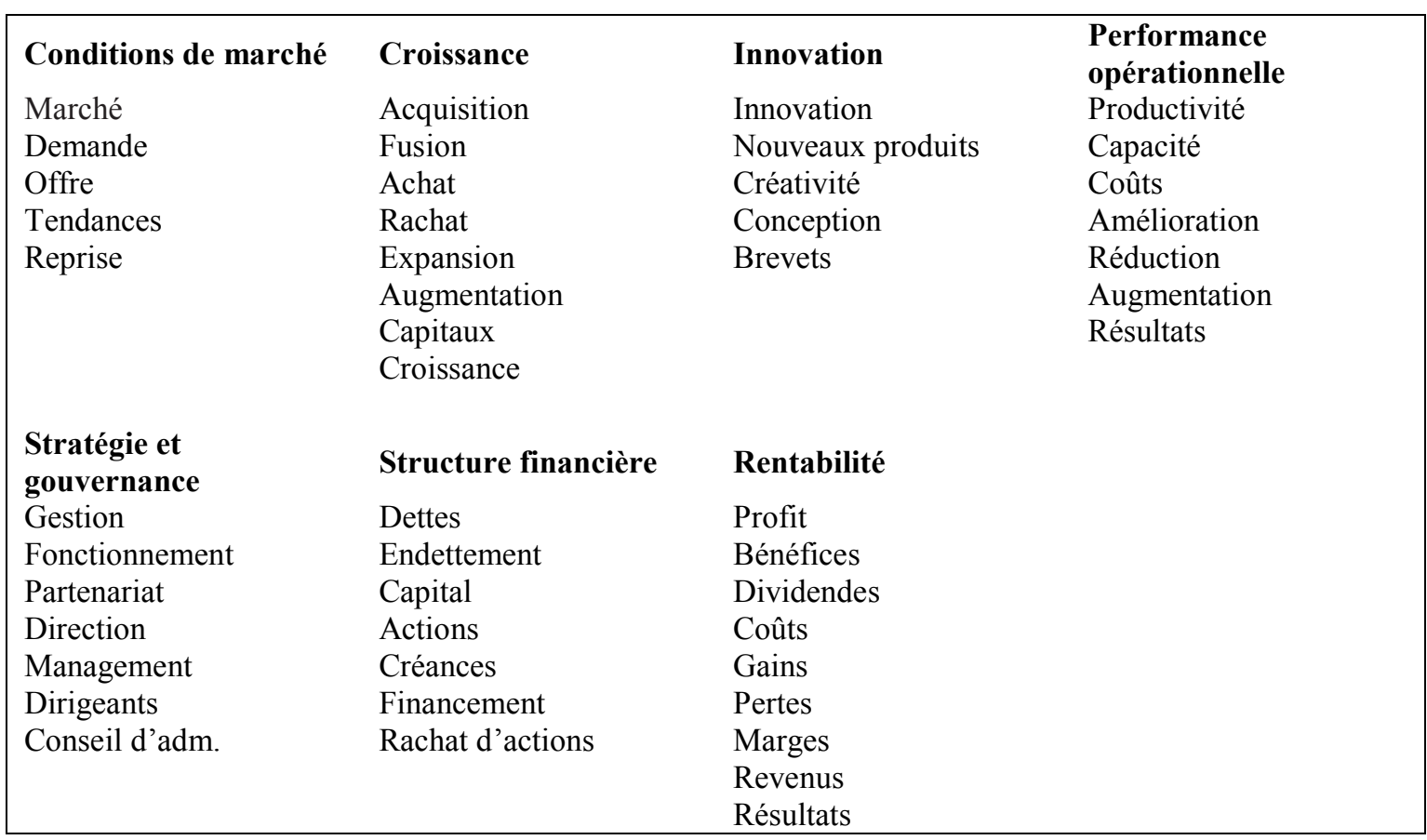

Des codes émergents non prévus initialement ont évidemment été pris en compte. Une fois les fréquences et cooccurrences observées, nous avons prévu un regroupement de ces codes en deux principaux super-codes: indicateurs financiers et indicateurs non financiers. De prime abord, avant détection de codes émergents, la rentabilité, la croissance de l'entreprise et la structure financière

\section{RÉSULTATS}

L'analyse de contenu des notes de recommandations des analystes et des entrevues transcrites a porté sur des codes représentatifs de la dimension financière et de la dimension non financière des indicateurs déterminants pour une recommandation d'achat, de maintien ou de vente de titre. Pendant la codification, des codes émergents ont été repérés, et le plus important d'entre eux, que nous avons nommé «Économie» regroupe les font figure d'indicateurs financiers tandis que les conditions de marché, la stratégie, l'innovation et la performance opérationnelle rentrent dans la case des indicateurs non financiers. Un rapprochement comparatif des deux groupes est donc indiqué pour révéler le poids relatif des indicateurs non financiers dans les choix de recommandation des analystes financiers.

motifs macroéconomiques à dimension nationale, internationale ou sectorielle qui ont fortement pesé dans des recommandations au sujet de titres.

Initialement ignorée à dessein de n'opposer que les éléments financiers et non financiers liés aux compagnies, cette dimension est finalement intégrée comme faisant partie des éléments non financiers sur lesquels l'entreprise n'a pas 
d'emprise. Force étant de constater que ce code «Économie» fait quasiment jeu égal avec le principal indicateur de performance financière qu'est la rentabilité. Nous maintenons sous la bannière «indicateurs non financiers » les informations non financières portant sur la performance organisationnelle, sur lesquelles les dirigeants ont un certain contrôle. Il s'agit notamment de l'innovation, la satisfaction de la clientèle, la compétence des employés et les processus internes, entre autres.

Les tableaux ci-dessous présentent le sommaire des scores en termes de mots et de citations pour chacun des codes.

Tableau 2 - Scores des codes - fréquences des citations

\begin{tabular}{|c|c|c|c|c|c|}
\hline & 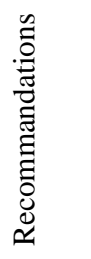 & 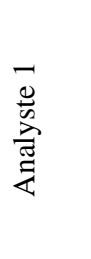 & 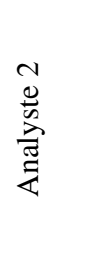 & 吾 & bo \\
\hline Conditions de marché & 11 & 2 & 8 & 21 & $10 \%$ \\
\hline Croissance & 19 & 5 & 1 & 25 & $12 \%$ \\
\hline Économie & 29 & 10 & 9 & 48 & $23 \%$ \\
\hline Innovation & 6 & 2 & 7 & 15 & $7 \%$ \\
\hline Performance & 11 & 3 & 2 & 16 & $8 \%$ \\
\hline Rentabilité & 35 & 9 & 9 & 53 & $25 \%$ \\
\hline Stratégie et gouvernance & 3 & 4 & 2 & 9 & $4 \%$ \\
\hline Structure financière & 4 & 4 & 5 & 13 & $6 \%$ \\
\hline Valeur client et part de marché & 8 & 1 & 4 & 13 & $6 \%$ \\
\hline TOTAL & 126 & 40 & 47 & 213 & $100 \%$ \\
\hline
\end{tabular}

Tableau 3 - Scores des codes - fréquences des mots

\begin{tabular}{|c|c|c|c|c|c|}
\hline & 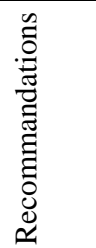 & 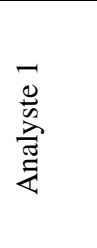 & 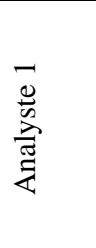 & 离 & $\delta^{\circ}$ \\
\hline Conditions de marché & 523 & 71 & 263 & 857 & $10 \%$ \\
\hline Croissance & 579 & 231 & 56 & 866 & $10 \%$ \\
\hline Économie & 1308 & 346 & 363 & 2017 & $24 \%$ \\
\hline Innovation & 274 & 105 & 281 & 660 & $8 \%$ \\
\hline Performance opérationnelle & 422 & 84 & 77 & 583 & $7 \%$ \\
\hline Rentabilité & 1451 & 312 & 284 & 2047 & $25 \%$ \\
\hline Stratégie et gouvernance & 132 & 83 & 62 & 277 & $3 \%$ \\
\hline Structure financière & 117 & 159 & 246 & 522 & $6 \%$ \\
\hline Valeur client et part de marché & 287 & 47 & 101 & 435 & $5 \%$ \\
\hline TOTAL & 5093 & 1438 & 1733 & 8264 & $100 \%$ \\
\hline
\end{tabular}

La rentabilité reste sans doute le premier élément $(25 \%)$ qui motive la recommandation des titres d'une entreprise, et qui ipso facto, améliore sa valeur marchande. Suivent alors les déterminants macroéconomiques, les perspectives de croissance et les conditions de marché. L'innovation et la performance opérationnelle sont les indicateurs non financiers corporatifs qui arrivent en premier, avec $8 \%$ et $7 \%$ de capacité de détermination d'une recommandation. Le meilleur score de l'innovation au sein des mesures non financières conforte la significativité observée pour cette variable dans la réduction de l'asymétrie d'information, dans certaines études quantitatives. C'est notamment un point sur lequel les répondants insistent. 
«[...] Idées, restructuration, cycle pour sa technologie, les ressources. Vraiment, il faut voir au niveau macroéconomique, et regarder à l'intérieur de la compagnie, s'il y a quelque chose de nouveau. Un jour c'est Research in Motion, et après c'est Apple avec son Iphone, ça bouge beaucoup en technologie.» (Analyste 2)

L'innovation est d'ailleurs l'élément qui avait prévalu lors de la croissance de la bulle financière dans le domaine des High-tech. Toutefois, il est question actuellement de s'assurer que l'innovation prise en compte n'exclut pas une distribution de dividendes; et cela permet d'ajuster le jugement des analystes afin de ne pas retomber dans l'excès d'optimisme qui fut à l'origine de la bulle.

Les analystes insistent sur le fait qu'il faut regarder ce qui se passe dans la compagnie. Ce qui soutient que les données non financières retenues et intégrées dans leur analyse sont davantage celles qui sont tournées vers la performance organisationnelle.
«[...] Il y a un très bon exemple, si vous vous souvenez d'Apple, tout le monde pensait qu'ils feraient faillite et que Microsoft les achèterait. Aujourd'hui ils se sont complètement réinventés. C'est une nouvelle compagnie. Ce n'est donc pas nécessairement cette structure qu'on suit en général : croissance, maturité, déclin. Il faut regarder ce qui se passe à l'intérieur, quelque chose qui peut faire en sorte qu'elle perdure. » (Analyste 2)

Trois super-codes ont été créés pour agréger les codes se rapportant au même axe d'analyse. Ainsi, le super-code Indicateurs économiques regroupe les codes Économie et Condition de marché. Il s'agit en fait de données non financières externes à l'entreprise et habituellement hors de son contrôle. Le super-code Indicateurs financiers associe les codes Croissance, Rentabilité et Structure financière. Enfin, le super-code Indicateurs non financiers reprend les codes Innovation, Gouvernance et stratégie, Performance, et Valeur client - part de marché. Le tableau présenté ci-dessous dessine les proportions d'importance des trois super-codes créés.

Tableau 4 - Distribution des super-codes

\begin{tabular}{|c|c|c|c|c|c|}
\hline & 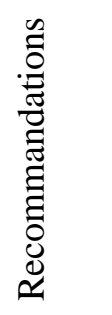 & 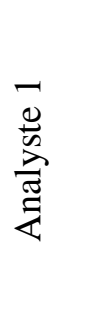 & 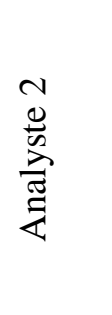 & 趈 & bo \\
\hline Conditions de marché & 11 & 2 & 8 & 21 & $10 \%$ \\
\hline Économie & 29 & 10 & 9 & 48 & $23 \%$ \\
\hline Sc. Indicateurs économiques & 40 & 12 & 17 & 69 & $32 \%$ \\
\hline Croissance & 19 & 5 & 1 & 25 & $12 \%$ \\
\hline Rentabilité & 35 & 9 & 9 & 53 & $25 \%$ \\
\hline Structure financière & 4 & 4 & 5 & 13 & $6 \%$ \\
\hline Sc. Indicateurs financiers & 58 & 18 & 15 & 91 & $43 \%$ \\
\hline Innovation & 6 & 2 & 7 & 15 & $7 \%$ \\
\hline Performance & 11 & 3 & 2 & 16 & $8 \%$ \\
\hline Stratégie et gouvernance & 3 & 4 & 2 & 9 & $4 \%$ \\
\hline Valeur client et part de marché & 8 & 1 & 4 & 13 & $6 \%$ \\
\hline Sc. Indicateurs non financiers & 28 & $\mathbf{1 0}$ & 15 & 53 & $25 \%$ \\
\hline Grand total & & & & & $100 \%$ \\
\hline
\end{tabular}


Les indicateurs financiers restent les plus importants (43\%), mais les scores des indicateurs non financiers, mis ensemble, occupent une part non négligeable ( $25 \%$ ), qui confirme bien leur portée.

Naturellement, les indicateurs financiers restent les plus importants $(43 \%)$, mais les scores des indicateurs non financiers, mis ensemble, occupent une part non négligeable ( $25 \%$ ), qui confirme bien leur portée, telle que décrite par bien des études antérieures ${ }^{1}$

Par ailleurs, un rapprochement des codes et supercodes montre nettement que l'écrasante majorité des arguments économiques et non financiers mis en avant pour justifier ou pour appuyer des recommandations, sont ceux qui présagent un impact direct ou à court terme sur la performance financière, et notamment la rentabilité; comme l'illustrent les extraits suivants :

\section{Économie}

Le marché européen est toutefois plus mal en point, note Jason Kupferberg, analyste d'UBS Investment Research, qui se demande si l'entreprise montréalaise ne publiera pas de moins bons résultats pour les prochains trimestres. $\mathrm{M}$. Steep et M. Kupferberg établissent un cours-cible de 18 \$ et 16 \$, respectivement. S.R. [1:44]

La réaction de la concurrence au lancement de Chat.r et l'arrivée de Vidéotron dans le domaine de la téléphonie sans fil au Québec font croire à Warren Hastings, analyste de Scotia Capitaux, qu'il y aura un ralentissement de la croissance au prochain trimestre. [1:38]

\section{Innovation}

Le nouveau BlackBerry Torch que l'entreprise a dévoilé mardi à New York a définitivement plu à Gus Papageorgiou, de Scotia Capitaux. Selon lui, il s'agit du meilleur appareil de RIM à ce jour et avec l'aide d'AT\&T pour la mise en marché aux ÉtatsUnis, le nouveau système d'exploitation et les nouvelles applications possibles, les ventes seront au rendez-vous, assure-t-il. Il maintient sa cote de «surperformance » et son prix cible à $117 \$$ pour le titre inscrit à Toronto. $[1: 2]$
L'entreprise fait preuve de créativité, souligne également M. Lacroix en montrant du doigt le lancement le 18 mai dernier par Cascades d'un essuie-main antibactérien qui «pourrait représenter une avenue de croissance significative pour le groupe ». [1:96]

\section{Performance opérationnelle}

«Puisqu'il n'y a pas d'arrêts de production prévus et que les prix de vente moyens seront plus élevés, nous croyons que le troisième trimestre sera encore plus fort que le deuxième », explique-t-il en maintenant sa cible à 125 \$ US. M.-C.M. [1 : 15]

\section{Gouvernance et stratégie}

Sa conversion en société par actions début 2011 constitue une mauvaise nouvelle pour les investisseurs, croit Bert Powell, analyste de BMO Marchés des capitaux. Selon lui, la nouvelle structure de l'entreprise et l'amortissement de l'acquisition de quatre firmes d'ingénierie auront un impact négatif sur les résultats en 2011. En conséquence, il rabaisse son prix cible de 31 à 28 \$ et ramène sa recommandation à «performance de secteur ». [1:98]

\section{Valeur client et parts de marché}

Plus difficiles, la baisse de confiance des consommateurs et la fin des crédits d'impôts à la rénovation, écrit $M$. Shreedhar. Modifiant légèrement ses prévisions, il diminue sa cible de 18 à 17 \$. M.-C.M. [1 : 4]

Jason Body, analyste de Scotia Capitaux, quant à lui, abaisse ses prévisions pour la même période. Le fait qu'Apple peine à répondre à la forte demande d'iPad aura un impact négatif sur la marge de profit, selon lui. [1 : 69]

Question : La satisfaction de la clientèle?

Analyste 2 : Extrêmement important!

Question : Les parts de marché?

Analyste 2: C'est aussi important parce qu'en général ça nous donne une bonne idée d'où la compagnie ira dans le futur. [2 : 91-94]

Ceci démontre bien que l'intérêt d'une recommandation et par ricochet, la valeur d'une entreprise, sont fortement liés à la capacité qu'a celle-ci à 
maintenir une excellente rentabilité, afin de fournir un rendement constant ou croissant aux investisseurs. Tous les analystes interviewés s'accordent d'ailleurs sur cette dimension comme étant primordiale pour leur analyse et leur recommandation, comme le témoignent les extraits suivants :

Analyste $1:$ « Je regarde la solidité financière de la compagnie, la sustainabilité du dividende qui est versé. »

Analyste 2: «Une chose par exemple, c'est le volume sur le marché. Les compagnies qui versent les dividendes, comme ça on tombe dans la grande capitalisation en général. »

\section{Le capital humain et intellectuel semble moins intéressant comme donnée pour les analystes financiers.}

En outre, le capital humain et intellectuel semble moins intéressant comme donnée pour les analystes financiers. Cet indicateur n'apparait quasiment pas dans les arguments qui soutiennent les recommandations, et les analystes interrogés affirment ne pas en tenir compte, en partie parce qu'ils ont difficilement accès à ce type d'informations, et ne peuvent véritablement pas l'évaluer d'eux-mêmes.

Un point sur lequel nos résultats ne corroborent pas ceux des études antérieures portant sur l'impact de la performance sociale et environnementale sur la performance boursière, est la responsabilité sociale et environnementale de l'entreprise. Alors que le discours dominant actuel indique la montée d'un investissement socialement responsable, la responsabilité sociale et environnementale de l'entreprise est quasi absente des documents que nous avons analysés, et les analystes interrogés affirment fermement ne pas en tenir compte, comme le montrent ces extraits :

Alors que le discours dominant actuel indique la montée d'un investissement socialement responsable, la responsabilité sociale et environnementale de l'entreprise est quasi absente des documents que nous avons analysés, et les analystes interrogés affirment fermement ne pas en tenir compte.
«C'est bien qu'une compagnie soit socialement responsable, mais à la fin de la journée, il faut voir si ça rapporte ou non. Souvent ça vaut la peine pour la compagnie, souvent non, la plupart des compagnies qui font de la responsabilité sociale et environnementale, c'est pour du Marketing. Elles ont toujours été socialement responsables ou alors, le type d'entreprise fait que ce soit socialement responsable. Je prends l'exemple de Suncor, qui est considérée comme socialement responsable. Sa cote de crédit en responsabilité sociale est très élevée, mais c'est une compagnie pétrolière, qui exploite des sables bitumineux, et qui n'est donc probablement pas bonne pour l'environnement, mais qui est bien cotée grâce à ses programmes implantés pour essayer d'améliorer l'environnement. Mais le coût que ça représente pour eux ne peut pas affecter leurs résultats. Ils veulent juste être perçus comme une compagnie socialement respon-sable, et ça les aide aussi pour les contrats avec les gouvernements. Surtout ces jours-ci, c'est toujours mieux d'être ou de paraître socialement responsable... cela ne m'affecte presque pas. »

(Analyste 1)

Il y a des gens qui la demandent [La RSE], mais c'est très peu. Les gens viennent pour que je protège leur capital et que je fasse des rendements. En général, $99 \%$ n'ont pas un intérêt social si ça va couper le rendement. Malheureusement, ce n'est pas quelque chose qui est important dans le monde d'argent.

Je peux vous dire que j'ai acheté BP pour des clients dernièrement. Car c'est une action qui revient fortement. Elle a baissé à cause du problème du pétrole, et mon job à moi c'est de faire de l'argent, ce n'est pas de sauver le monde. Malheureusement, dans le monde d'argent, la responsabilité sociale est peu intéressante. (Analyste 2)

Avec la révolution écologique, la RSE a pris une nouvelle dimension et intéresse davantage les médias, lesquels sensibilisent un plus grand public. Les entreprises en sont conscientes et adoptent une série de mesures et des politiques de commu- 
nication pour préserver, acquérir, maintenir ou restaurer leur légitimité, indépendamment de leur réelle performance en la matière. C'est cette légitimité qui leur permet de maintenir une certaine opinion publique favorable à leurs produits et services. Sous cette optique, même si la RSE semble ne pas avoir un impact direct sur la rentabilité, elle y concourt tout de même en préservant les activités de la compagnie des attaques et boycotts éventuels que pourraient commanditer des groupes de pression non satisfaits

\section{CONCLUSION ET CONTRIBUTIONS}

\section{Les acteurs de marché, les analystes \\ financiers en particulier, montrent un intérêt grandissant pour des informations non financières et plus précisément celles qui donnent une idée de la performance organisationnelle des entreprises.}

L'information financière qui est par ailleurs difficilement compréhensible par certains acteurs des marchés financiers et certaines parties prenantes aux activités de l'entreprise a montré des limites quant à sa capacité à refléter toutes les dimensions des entreprises et à conférer une précision aux prévisions de cours. Les acteurs de marché, les analystes financiers en particulier, montrent un intérêt grandissant pour des informations non financières et plus précisément celles qui donnent une idée de la performance organisationnelle des entreprises. L'efficacité du comportement de l'analyste étant toute aussi une question de compétences que de mesures d'incitation, elle peut être affectée par leurs attentes au sujet des informations, de même que par l'offre spécifique d'informations par l'entreprise (Pope, 2003). Les stratégies de divulgation des entreprises devraient également tenir compte de ce que les analystes financiers ne prennent en considération que des informations non financières qui leur permettent de prédire la performance financière à court terme.

Des études antérieures ont déjà indiqué l'intérêt grandissant pour l'information non financière. Cette étude ambitionne alors de les compléter en expliquant de quelle manière ces informations non de sa politique sociale et environnementale. D'emblée, les analystes ne tiennent pas compte de la RSE, mais celle-ci agit tout de même par ricochet sur la performance financière et boursière de la compagnie. Car, une chute des ventes due au boycott d'un produit serait sans doute prise en compte par des analystes. Et si ce boycott est lié aux problèmes sociaux environnementaux, la RSE sera ainsi prise en compte en filigrane. Les analystes semblent donc en tenir compte à l'insu de leur plein gré

financières sont prises en compte dans le processus décisionnel qui mène à un investissement au sein d'une firme, et comment elles contribuent à déterminer la valeur marchande de celle-ci.

\section{BIBLIOGRAPHIE}

${ }^{1}$ Amir, E et Lev B., Value-relevance of Nonfinancial Information: The Wireless Communications Industry, Journal of Accounting \& Economics, 22 (AugustDecember 1996): 3-30. Cormier, D., Aerts, W., Ledoux, M-J., et Magnan, M., Attributes of Social and Human Capital Disclosure and Information Asymmetry between Managers and Investors, Canadian Journal of Administrative Sciences, 2008 (soumis) Graham, C.M., M.V. Cannice et T.L. Sayre, The value relevance of financial and non- information for internet companies, Thunderbird International Business Review 44 (1) (2002). pp. 47-70. Ittner, C. D., et D. Larcker (1998). Are nonfinancial measures leading indicators of financial performance? An analysis of customer satisfaction, Journal of Accounting Research Pope, P., Discussion of Disclosure Practices, Enforcement of Accounting Standards, Analysts' Forecast Accuracy : An International Study, Journal of Accounting Research, Volume 41 Issue 2, April 2003, Pages 273 - 283. 


\section{FORMULAIRE D'ABONNEMENT}

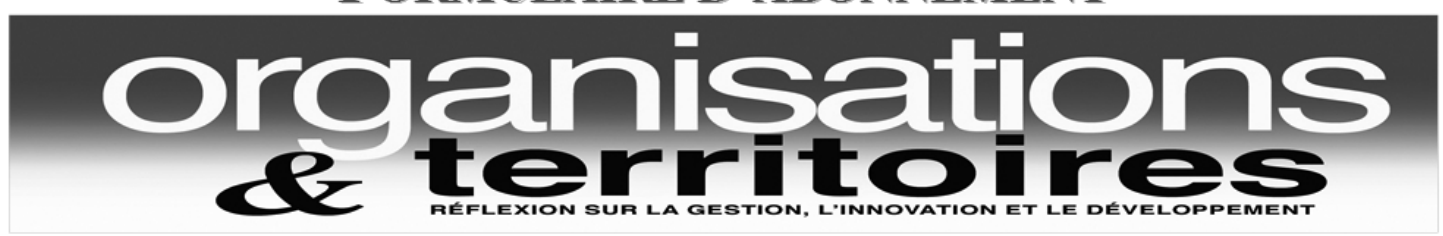

Je désire m'abonner à la revue Organisations et territoires et je joins un chèque au montant de :

Nouvel abonné $(\sqrt{ }):$

Renouvellement $(\sqrt{ }):$

Date :
Abonnement institutionnel
(6 numéros)
$103,48 \mathrm{~S}$
(Toutes taxes incluses)
Abonnement individuel
(6 numéros)
$66,69 \$$
(Toutes taxes incluses)
Abonnement étudiant
(6 numéros)
$48,29 \$$
(Tx inc.) (Envoyer photocopie carte étud.)
Abonnement gouvernemental (6 numéros)
$90,00 \$$
(Fournir certificat d'exemption des taxes)
Abonnement hors Canada
(6 numéros) $145,00 \$$
$(110 \$+35 \$$ frais de poste) (sans taxes)

Tous les abonnements sont d'une durée de deux ans

- J'aimerais que mon abonnement débute avec le DERNIER numéro paru, soit :

Volume $\mathrm{N}^{\circ}$

- J'aimerais que mon abonnement débute avec le PROCHAIN numéro à paraître, soit :

Volume $\mathrm{N}^{\circ}$

\section{Faites-nous parvenir vos coordonnées :}

Nom :

Organisation :

Adresse :

Téléphone :

Télécopieur :

Courriel :

Adresse d'expédition :

Revue Organisations et territoires

Université du Québec à Chicoutimi

555, boulevard de l'Université, bureau H6-1430

Chicoutimi (Québec) CANADA G7H 2B1

Téléphone : (418) 545-5011, poste 4530 Télécopieur : (418) 693-9072

Courriel : revueot@uqac.ca Site Web : www.uqac.ca/revueot 